\title{
Opposition Based Teaching Learning Optimization For Combined Economic Emission Dispatch Problem
}

\author{
${ }^{1}$ D. Sai Krishna Kanth, ${ }^{2} S$ S Deekshit, ${ }^{3} M$ G Mahesh \\ ${ }^{1,2,3}$ Dept. of EEE, Annamacharya Institute of Technology and Sciences, Rajampet, AP, India \\ saikrishnakanth.dskk@gmail.com, deekshitkoushika@gmail.com, mahi.leo5611@gmail.com
}

\begin{abstract}
Cost minimization of power generation is one of the most important powers for generation in a power system. The aim of this Project is to find the optimum set of power to be generated for a given loading conditions. In this Project, a new algorithm opposition based teaching learning based optimization is developed and applied to ED problem. The proposed OTLBO do not have the drawbacks of the classical heuristics such as local optimal trapping due to premature convergence, insufficient capability to find nearby extreme points, and lack of efficient mechanism to treat the constraints. The algorithm describes two basic modes of the learning: (i) through teacher (known as teacher phase) and (ii) interacting with the other learners (known as learner phase). The effectiveness and feasibility of the proposed OTLBO method were demonstrated on 6 and 10-unit test systems and then compared with other algorithms like PSO and HSA. The experiments showed that the proposed approach was capable of determining higher quality solution while addressing the complex ED problems.
\end{abstract}

Index Terms-Economic Dispatch; Teaching Learning Based Optimization; Application of OTLBO on Economic Dispatch; Comparison of OTLBO with Other Algorithms

\section{I.INTRODUCTION}

A meta-heuristic is strictly described as an iterative generation method which publications a subordinate heuristic thru merging perceptively precise ideas for exploring and exploiting the hunt place, studying techniques are used to form data on the way to find successfully close to-maximum dependable solutions. Therefore optimization techniques had been broadly applied in energy machine operation. Many mathematical methods together with lambda new release method, gradient approach and quadratic programming approach [1] carried out for solving ED trouble with the aid of assuming that the incremental fee curve as piecewise-linear monotonically growing feature. M. Basu [2] presented incorporated evolutionary programming; tabs seek (TS) and Quadratic programming (QP) techniques to resolve non-convex ED troubles. This blanketed artificial intelligence method moreover calls for -section computations.

Several mathematical programming along with linear and non-linear programming were beneficial for solving convex ED troubles. The conventional algorithms, dynamic programming enacts no regulations on the nature of the fee curve and therefore it is able to remedy ED issues. But, this technique can't be implemented to remedy large dimensional issues because of requirement of large computational efforts. A number of the famous metaheuristics advanced over the past Three a long term are: Genetic set of rules (GA) [1] which fits on the principle of

device of the character; Ant Colony Optimization (ACO) [3] which goes at the looking behaviour of the ant for the food; Particle Swarm Optimization (PSO) [four] which is going on the looking behaviour of the swarm of birds; Differential Evolution (DE) [4] that is similar to GA with specialized crossover and preference technique; harmony are looking for (HS) [5] which goes on the precept of track inventiveness in a track player; microorganism Foraging Optimization (BFO) [6] which goes on the behaviour of micro-organism; Shuffled Frog leaping (SFL) [nine] which fits at the precept of verbal exchange a number of the frogs; Shuffled Differential Evolution (SDE) which suits just like DE with quantified crossover and choice approach; changed Shuffled Frog jumping set of guidelines (SFLA) which fits at the principle of verbal exchange amongst frogs; Biogeography-primarily based completely Optimization (BBO) which suits on the precept of migration and relocation of the species from one place to the opportunity; solving most useful electricity go along with the go with the flow issues using bacterial swarm optimization set of rules proposed in which goes on load waft research; those algorithms were completed to many engineering optimization troubles and proved active to remedy a few precise form of troubles. Multi-objective evolutionary algorithm for mixed economic Emission Dispatch trouble is proposed in [7] on this method the financial dispatch hassle is formulated as a nonlinear constrained multi-goal optimization problem and evolutionary set of rules is developed to replace the economic Emission Dispatch. The principle aim of the financial dispatch is to lessen the technology rate. O. AL sac furnished perfect load waft with consistent-state protection in [8].

\section{FORMULATION OF ECONOMIC DISPATCH PROBLEM}

Generally economic dispatch is defined as the process of allocating generation levels to the generating units, so that load is supplied entirely and most economically. In an electrical power system, economic dispatch is the one of the most important optimization problem and it is a crucial task in the economic operation. The main objective of this economic dispatch problem is to determine the optimal combination of power outputs for all generating units that minimizes the total fuel cost while satisfying load demand and operating constraints that are equality and inequality constraints. 


\section{A. ED Problem with Smooth Cost Function}

The ED problem is to locate the surest combination of strength generations that minimizes the full technology cost even as gratifying an equality constraint and inequality constraints.

The most simplified fee feature of each generator may be represented as Quadratic function as given in whose answer may be acquired by way of the conventional mathematical strategies

$$
\begin{array}{cc}
\boldsymbol{F}_{i} \boldsymbol{P}_{i}=\boldsymbol{a}_{i} \boldsymbol{P}_{i}+\boldsymbol{b}_{i} \boldsymbol{P}_{i}+\boldsymbol{c}_{i} & \ldots .2 \\
\text { Where } & \text { Total fuel cost } \\
\text { Fuel cost function of generator i } & \\
\text { Fuel cost coefficients of generator i } & \text { Electrical output of generator i } \\
\boldsymbol{N} \quad \text { Total number of generators }
\end{array}
$$

While minimizing the total generation cost, the generation should satisfy the following constraints.

\section{B. Equality Constraint}

While minimizing the total generation cost, the total generation should be equal to the total demand plus the network transmission losses.

$$
\sum_{i=1}^{W} P_{i}-
$$

Where $P_{D}$ is the total system demand and $\mathrm{P}_{L}$ is the transmission line loss. In this paper transmission line losses are not considered.

\section{Inequality Constraint}

The generation output of each unit should be between its minimum and maximum limits.

$$
\begin{gathered}
\text { Pimin } \leq P i \leq \text { Pimax } \\
\text { Where Pimin }
\end{gathered}
$$

min and max output of generator $\mathrm{i}$.

\section{PROPOSED METHOD}

Opposition Based Teaching Learning Optimization

$$
\text { Algorithm }
$$

The concept of competition-primarily based analysing (OBL) have become brought via the use of Tizhoosh and has thus far been completed to reinforce up reinforcement studying and back propagation learning in neural networks [9]. The precept concept behind OBL is the simultaneous attention of an estimate and its corresponding opposite estimate (i.e., wager and contrary bet) which will advantage a better approximation for the contemporary learner answer. In this paper, OBL has been carried out to reinforce up the convergence price of TLO. For this reason, our proposed approach has been referred to as competition-based absolutely training gaining knowledge of based absolutely optimization (OBTLO). OBTLO makes use of opposite numbers in the course of populace initialization and additionally for generating new populations at some stage in the evolutionary procedure.
To the amazing of our know-how, this is the primary time that contrary numbers were utilized to hurry up the convergence fee of this optimization algorithm.

\section{OPPOSITION BASED LEARNING}

Typically talking, evolutionary optimizations strategies begin with some initial solutions (preliminary population) and attempt to enhance them toward some most appropriate solution(s). The manner of searching terminates while a few predefined criteria are happy. In the absence of a priori facts approximately the answer, we usually start with random guesses. The computation time, amongst others, is related to the space of those preliminary guesses from the greatest answer. We are capable of enhance our danger of beginning with a more in-depth (healthier) solution by concurrently checking the opportunity solution. By using doing this, the more fit one (bet or contrary wager) can be decided on as a preliminary solution. In truth, constant with risk idea, $50 \%$ of the time a wager is in addition from the answer than its contrary guess. Consequently, beginning with the closer of the 2 guesses (as judged with the resource of its health) has the potential to reinforce up convergence. The identical method can be carried out no longer handiest to initial solutions however additionally continuously to every solution inside the contemporary populace. However, in advance than focusing on OBL, we want to define the concept of contrary numbers [10].

Similar to all populace-primarily based optimization algorithms, important steps are distinguishable for TLBO, particularly, populace initialization and producing new generations via evolutionary operations consisting of teaching segment and getting to know segment. we can beautify those two steps using the OBL scheme. The authentic TLBO is selected as a parent algorithm and the proposed opposition-based totally thoughts are embedded in TLBO to boost up its convergence speed.

\section{A. OPPOSITION BASED POPULATION INITIALIZATION}

Regular with our evaluation of optimization literature, random quantity generation, in absence of a priori information, is the not unusual desire to create an initial populace. Consequently, as noted in phase 2.B, with the resource of the usage of OBL, we are capable of achieve more healthy beginning learner answers even though there's no a priori statistics about the answer(s). The subsequent steps gift that procedure

1) Initialize the population Pn randomly

2) Calculate the opposite population by

$$
O P_{i_{i j} j}=a_{j}+b_{j}-P_{i_{j} j}
$$

Where $P i, j$ and $O P i, \mathrm{j}$ denote $\mathrm{j}$ Th variable of the $i$ th vector of the population and the opposite Population, respectively.

3) select the Pn fittest individuals from $\{P \cup O P\}$ as initial population.

\section{B. OPPOSITION BASED GENERATION JUMPING \\ Via applying a comparable approach to the current population, the evolutionary method may be}


compelled to leap to a brand new learner answer, which preferably is healthier than the cutting-edge one. Primarily based definitely on a jumping price $\mathbf{J} \mathrm{r}$ (i.e., jumping threat), after generating new populations via training section and getting to know segment, the opportunity population is calculated and the Pn fittest

Individuals are decided on from the union of the present populace and the alternative population. In evaluation to competition based initialization, generation jumping calculates the opposite populace dynamically. In place of the use of variables predefined c program language duration limitations ([aj bj]), technology jumping calculates the other of every variable based totally on minimal $\left(\min _{p}^{j}\right)$ and most $\left(\max _{p}^{j}\right)$ values of that variable in the cutting-edge population

Human beings are decided on from the union of the winning populace and the opportunity population. No longer like opposition primarily based definitely initialization, technology leaping calculates the alternative populace dynamically. Instead of the use of variables predefined c programming language obstacles ([aj bj]), era leaping calculates the alternative of each variable based totally on minimum $\left(\min _{p}^{j}\right)$ and most $\left(\min _{p}^{j}\right)$ values of that variable inside the contemporary population

$$
O P_{\mathrm{i}_{j} j}=\min _{\mathrm{p}}^{j}+\max _{\mathrm{p}}^{j}-p_{\mathrm{i}_{\mathrm{j}} j}
$$

Where $\quad i=1,2 \ldots p n$ and $j=1,2 \ldots$. .

By continuing within variables interval static boundaries, we would jump outside of the already shrunken search class and the knowledge of the current congregated population would be lost. Hence, we calculate opposite points by using variables current interval in the population $\left[\min _{p}^{j}, \max _{p}^{j}\right]$ which is, as the search does progress, gradually smaller than the corresponding initial range [ai,bj].

\section{APPLICATION OF OTLBO FOR ED PROBLEM}

The step-wise procedure for the implementation of OTLBO is given in this section.

Step-1: Define the optimization problem and initialize the optimization parameters. Initialize the population size (Pn), number of generations (Gn), number of design variables (Dn), and limits of design variables (UL, LL).

Define the optimization problem as: minimize $f(X)$

Subject to $\mathrm{Xi}$ belongs to $\mathrm{xi}=1,2 \ldots \mathrm{Dn}$

Where $f(X)$ is the objective function, $X$ is a vector for design variables such that

$$
\mathrm{L}_{\mathrm{i}_{i} j} \leq X_{\mathrm{i}} \leq U_{\mathrm{i}_{0} j}
$$

Step-2: Initialize the population

Generate a random populace in step with the population length and variety of variables. For TLBO, the population length shows the range of novices and the design variables indicates variety of topics supplied. This population is expressed as

$$
\text { Population }=\left[\begin{array}{ccc}
P_{1,1} & \cdots & P_{1_{j}, D n} \\
\vdots & \cdots & \vdots \\
P_{\mathrm{pn}_{1} 1} & \cdots & P_{\mathrm{pn}, D n}
\end{array}\right]
$$

Step-3: Initialize opposition based population Calculate opposite population by

$$
\begin{aligned}
& \quad O P_{i_{i j} j}=U l_{j}-L l_{j}-P_{i_{i} j} \\
& i=1,2, \ldots, P_{n} \text { and } j=1,2, \ldots, D_{n}
\end{aligned}
$$

Where $\mathrm{Pi}, \mathrm{j}$ and $\mathrm{OPi}, \mathrm{j}$ denote $\mathrm{j}$ Th variable of the $\mathrm{i}$ th vector of the population and the opposite-population, respectively.

Step-4: Select the Pn fittest individuals from $\{P \cup O P\}$ as initial population.

Step-5: Teacher Phase.

Calculate the imply of the populace column-wise, on the way to supply the imply for the specific concern as M, D $=[\mathrm{m} 1, \mathrm{~m} 2 \ldots \mathrm{mD}]$.

The best solution will acts as a teacher for that iteration Xteacher $=\mathrm{Xf}(\mathrm{x})=\min$

The teacher will try to shift the mean from $\mathrm{mD}$ towards Xteacher which will act as a new mean for the iteration. So,

Mnew $=$ Xteacher

The difference between two means is expressed as Difference $=r *($ Mnew-TFMi $)$

The value of TF is selected as 1 or 2 . The obtained difference is added to the current solution to update its values using

$\mathrm{X}$ new, $\mathrm{i}=\mathrm{X}$ old, $\mathrm{i}+$ difference

Accept Xnew if it gives better function value.

Step-6: Learner Phase.

Freshmen increase their knowledge by means of one-of-akind ways: one through enter from the teacher. And the opposite via freshmen will increase their information by interaction among themselves. A learner interacts randomly with different rookies for reinforcing his or her know-how. A learner learns new matters if the alternative learner has extra know-how than him.

Here, we considering a population size of $n$, the learning phenomenon of this phase is expressed below.

Randomly select two learners $X i$ and $X j$ where $i \neq j$

$X$ new, $i=X o l d, i+r *(X i-X j)$ if $f(X i)<f(X j)$

Xnew,i=Xold, $i+r *(X j-X i)$ if $f(X i)>f(X j)$

Accept $X n e w, i$ if it gives better function value

Step-7: Opposition based generation jumping

General jumping calculates the opposite of each variable based on minimum $\left(L l_{j}^{p}\right)$ and maximum $\left(U l_{j}^{p}\right)$ values of that variable in the current population

Take some jumping rate $\mathrm{Jr}$ and then

OXnew $i, j=$ Ulj + Llj - Xnew $i, j \quad$ if rand $<\mathrm{Jr}$

OXnew $\mathrm{i}, \mathrm{j}=$ Xnew $\mathrm{i}, \mathrm{j} \quad$ if rand $>\mathrm{Jr}$

Step 8: Termination criterion.

Stop if maximum generation number is achieved; otherwise repeat from step 3 .

\section{COMPARISON OF OTLBO WITH OTHER ALGORITHMS}

The proposed strategies TLBO and OTLBO are simulated at wonderful goal functions and tabulate in desk four.2 and 4.three respectively. Evaluation of simulation effects for the proposed techniques is proven in table 4.3 as proven in desk four. Three, in case of price goal the price received thru TLBO is $111289.9814 \$ / \mathrm{hr}$ with emission and energy loss are 4422.7320ton/hr \& 87.1267MW respectively. The received charge, emission and strength loss thru OTLBO are $111261.5051 \$ / \mathrm{hr}$, 
4370.2634ton/hr and $87.0403 \mathrm{MW}$ respectively. The acquired fee, emission and power loss via OTLBO are $116121.5912 \$ / \mathrm{hr}, \quad 3831.5722 \mathrm{ton} / \mathrm{hr} \quad \& \quad$ eighty two. $2178 \mathrm{MW}$ respectively. Now in the case of mixed economic emission dispatch (CEED) the fee acquired by the use of TLBO is $112714.5403 \$ / \mathrm{hr}$ with emission and strength loss are 4019.6381ton/hr \& 84.9928MW respectively. The obtained price, emission and strength loss thru OTLBO are $112710.0059 \$ / \mathrm{hr}, 4019.0078 \mathrm{ton} / \mathrm{hr}$ and eighty five.0167MW respectively. From the consequences it can be saying that the better consequences are acquired by using the usage of OTLBO at the same time as compared with TLBO.

VI.SIMULATION RESULTS

Comparison of simulation results for 10-unit system with quadratic cost function

\begin{tabular}{|c|c|c|c|c|c|c|}
\hline & \multicolumn{2}{|c|}{$\begin{array}{c}\text { COST } \\
\text { OBJECTIVE }\end{array}$} & \multicolumn{2}{c|}{$\begin{array}{c}\text { EMMISSION } \\
\text { OBJECTIVE }\end{array}$} & \multicolumn{2}{c|}{ CEED } \\
\hline & TLBO & OTLBO & TLBO & OTLBO & TLBO & OTLBO \\
\hline $\begin{array}{c}\text { Cost in } \\
\$ / h r\end{array}$ & 11128 & 111261. & 11612 & 116121.6 & 11271 & 112710. \\
1 & 5 & 9.1 & & 4.5 & 1 \\
\hline $\begin{array}{c}\text { Emmissio } \\
\mathrm{n} \text { in ton/hr }\end{array}$ & 4422.7 & 4370.26 & 3831.8 & 3831.57 & 4019.6 & 4019.00 \\
\hline $\begin{array}{c}\text { Power loss } \\
\text { in MW }\end{array}$ & 87.12 & 87.0403 & 82.189 & 81.2178 & 84.992 & 84.0167 \\
\end{tabular}

\section{CONCLUSION}

Contemporary ED problem can be solved in extraordinary times with unique strategies. In first case ED hassle were considered with quadratic price characteristic, which always exists in energy structures. The proposed algorithms TLBO \& OTLBO have been efficaciously applied to remedy this ED trouble thinking about 10-unit check system and additionally in comparison with lambda new release technique to test the performance of the proposed set of rules. The proposed algorithm OTLBO determined better answer for all test systems than TLBO. The result genuinely suggests that the proposed approach can be used as an efficient optimizer supplying awesome solutions for financial dispatch problems.

In the subsequent case of the zero.33 chapter heuristic techniques which consist of TLBO \& OTLBO have been efficaciously implemented to resolve strength system ED problem. The opportunity, efficiency of the proposed algorithms has been investigated on strength machine having 10-unit tool. The simulation effects of three check systems indicates that proposed strategies succeeded in attaining the purpose of bargain in rate of era. Proposed set of regulations OTLBO received minimal fuel fee for all test systems, whilst compared with TLBO. The proposed technique notably enhances the searching functionality and efficiently manages the system constraints. The achievement optimizing performance at the validation data set illustrates the performance of the proposed approach and display that it may be used as a dependable device for ED hassle.

In fourth bankruptcy the optimization techniques particularly TLBO \& OTLBO were employed on the combined monetary and emission dispatch problem with quadratic and valve thing loading effect respectively. The proposed algorithms offer outstanding compromise solution in case of 10-unit test system. The set of rules gives higher maximum suitable solution than those acquired from different complicated algorithms. The consequences display that proposed technique is green for multi-goal optimization algorithm. hence the answer of monetary emission dispatch for the high-quality compromise answer out of many most super solutions over the change-off curve is classified and permits the power tool operator to alter the generation tiers effectively and efficaciously. it may be concluded that proposed techniques seem like a strong and reliable optimization set of rules for the answer of numerous multi-objective power device optimization problems.

\section{ACKNOWLEDGEMENT}

We would like to thank Mr. G. Sudha Kiran, Assistant Professor, ECE Department, Annamacharya Institute of Technology and Sciences, Rajampet.

\section{REFERENCES}

[1] S.Hemamalini, and shishaj p simon, Emission constrained economic dispatch with valve point effect using particle swarm optimization

[2] M. Basu Economic environmental dispatch using multiobjective differential evolution November 2010, Elsevier Ltd.

[3] Kamal K Mandal, N chakrabarthy, Effect of Control Parameters on Differential Evolution based Combined Economic Emission Dispatch with Valve-Point Loading and Transmission Loss. International journal of emerging electric power systems.

[4] M. sydulu,"A very fast and effective non-iterative $\lambda$ - logic based algorithm for economic dispatch of thermal units, Proceedings of EEE Tencon, 1999.

[5] A. Srinivasa Reddy, K Vaisakh, Economic Emission Load Dispatch by Modified Shuffled Frog Leaping Algorithm, International Journal of Computer Applications 31 (11), pp. 35-42.

[6] K. Vaisakh, A. Srinivasa Reddy,"MSFLA/GHS/SFLAGHS/ SDE algorithms for economic dispatch problems considering multiple fuels and valve point loadings", Applied soft computing 13(11), pp. 4281-4297.

[7] A. Srinivasa Reddy, K Vaisakh,"Environmental Constrained Economic Dispatch by Modified Shuffled Frog Leaping Algorithm", Journal of Bioinformatics and Intelligent Control 2 (3), pp. 216-222

[8] Rao RV, Savsani VJ, Vakharia DP.,"Teaching-learningbased optimization: A novel method for constrained mechanical design optimization problems", Comput Aided Des 2011;43: pp. 303-15.

[9] Rao RV, Savsani VJ, Vakharia DP. Teaching-learningbased optimization:an optimization method for continuous non-linear large scale problems. Inf Sci2012;183:1-15.

[10] R.Venkata Rao, Vivek Patel, An improved teaching learning based optimization algorithm for solving unconstrained optimization problems. October 2012, Elsevier Ltd. 